\title{
Metschnikowia pulcherrima
}

National Cancer Institute

\section{Source}

National Cancer Institute. Metschnikowia pulcherrima. NCI Thesaurus. Code C127731.

A species of yeast in the phylum Ascomycota commonly found on grapes, cherries,

flowers, and spoiled fruit. Each cell of M. pulcherrima contains a single globule of oil and the species is being investigated as a source for a palm oil alternative. 